\title{
Inverse Proportional Relationship Between Switching-Time Length and Fractal-Like Structure for Continuous Tracking Movement
}

\author{
Takehito Hirakawa \\ Physical Education Center, Nanzan University, \\ Nagoya 466-8673, Japan \\ takehito@nanzan-u.ac.jp \\ Hiroo Suzuki \\ Faculty of Economics, Ryukoku University, \\ Kyoto 612-8577, Japan \\ hsuzuki@econ.ryukoku.ac.jp \\ Kazutoshi Gohara \\ Division of Applied Physics, Hokkaido University, \\ Sapporo 060-8628, Japan \\ gohara@eng.hokudai.ac.jp \\ Yuji Yamamoto \\ Research Center of Health, Physical Fitness and Sports, \\ Nagoya University, Nagoya 464-8601, Japan \\ yamamoto@htc.nagoya-u.ac.jp
}

Received December 13, 2016; Revised August 16, 2017

\begin{abstract}
We investigate the relationship between the switching-time length $T$ and the fractal-like feature that characterizes the behavior of dissipative dynamical systems excited by external temporal inputs for tracking movement. Seven healthy right-handed male participants were asked to continuously track light-emitting diodes that were located on the right and left sides in front of them. These movements were performed under two conditions: when the same input pattern was repeated (the periodic-input condition) and when two different input patterns were switched stochastically (the switching-input condition). The repeated time lengths of input patterns during these conditions were $2.00,1.00,0.75,0.50,0.35$, and $0.25 \mathrm{~s}$. The movements of a lever held between a participant's thumb and index finger were measured by a motion-capture system and were analyzed with respect to position and velocity. The condition in which the same input was repeated revealed that two different stable trajectories existed in a cylindrical state space, while the condition in which the inputs were switched induced transitions between these two trajectories. These two different trajectories were considered as excited attractors. The transitions between the two excited attractors produced eight trajectories; they were then characterized by a fractal-like feature as a third-order sequence effect. Moreover, correlation dimensions, which are typically used to evaluate fractal-like features, calculated from the set on the Poincaré section increased as the switching-time length $T$ decreased. These results suggest that an inverse
\end{abstract}

This is an Open Access article published by World Scientific Publishing Company. It is distributed under the terms of the Creative Commons Attribution 4.0 (CC-BY) License. Further distribution of this work is permitted, provided the original work is properly cited. 
proportional relationship exists between the switching-time length $T$ and the fractal-like feature of human movement.

Keywords: Excited attractor; external temporal input; dynamical systems.

\section{Introduction}

The famous early works in which dynamical systems theory was applied to human movement were the experimental verification by Kelso [1984] and the mathematical model of Haken et al. [1985]. They examined the dynamic state of the relative phase between two limbs. Their main point of view is the phase transition from out-of-phase to in-phase states by intrinsic dvnamics STchöner \& Kelso, 1988], which is an internal human parameter. These phases are understood as attractors that are important for human motor control. Several studies by Kelso and his colleagues are considered to be based on an autonomous system or an autonomous dynamical system [Yamamoto \& Gohara, 2000] because of the closed system that is independent of other systems. These studies addressed tasks in which participants moved limbs periodically to metronome sounds that were changed gently, i.e. a control parameter. In contrast, a dynamical system, which is open to other systems, is considered a nonautonomous system or a nonautonomous dynamical system Gohara \& Okuvama, 1999a. In this system, movement patterns are excited by external inputs that are external parameters. An attractor, which is excited with an external input, underlies a movement pattern; i.e. this is considered an excited attractor. Recent studies based on this dynamical systems theory, for example not only the physical phenomenon Arecchi et al. 1986: Constantin et al., 1991; Maas et al., 1997; Matias et al., 1997; Mestl et al., 1997; Schmiegel \& Eckhardt, 1997; Tanii et al., 1999; Tanii et al., 1991] but also human movement Hirakawa et al.. 2016: Suzuki \& Yamamoto, 2015; Yamamoto \& Gohara, 2000], have revealed that the spatiotemporal structure in various natural phenomena is fractal-like and/or self-similar. These studies on human movement entailed tasks in which participants switched several different movement patterns continuously with the external inputs being abruptly switched.

This implies that, "for autonomous dynamical systems, the basic law of evolution is static in the sense that the environment does not change with time. In many applications, however, such a static approach is too restrictive and a temporally fluctuating environment must be taken into account" Kloeden \& Pötzsche, 2013. Consequently, "in reasonable models adapted to and well-studied for problems in temporally fluctuating environments, the evolutionary equations have to depend explicitly on time through time-dependent parameters or external inputs" Kloeden \& Pötzsche, 2013. Therefore, nonautonomous dynamical systems have been applied to biological systems, even life sciences widely, in recent years owing to the importance of utilization of dissipative dynamical systems theory. This perspective in their studies, based on nonautonomous dynamical systems, differs from that of the series of studies by Kelso mentioned above. Moreover, Nishikawa and Gohara 2008, 2008] established that we are able to quantify a state in a continuous dynamical system with a fractal dimension, although the fractals are generated by an iterated function system, e.g. the Sierpinski gasket, if we design an experiment by a theoretical framework for a continuous dynamical system stochastically excited by an external temporal input. In this study, we apply the hypothesis with a dissipative dynamical system by Gohara and Okuyama [1999a, 1999b], Nishikawa and Gohara [2008, 2008] to our experiment. This theoretical framework is detailed in the next section.

Figure 1 shows schematics highlighting the difference between autonomous and nonautonomous systems Hirakawa et al., 2016]. For instance, Figs. 1(a) and 1(b) show autonomous systems. Figure1(a) implies a state in which the same tracking movement pattern toward the left side (L) is repeatedly excited with temporal input in this

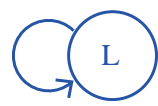

(a)

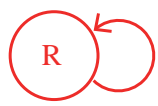

(b)

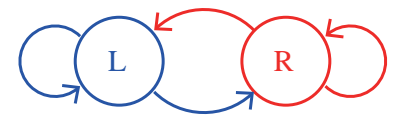

(c)
Fig. 1. Schematics of the interplay between systems Hirakawa et al., 2016. See the body of the text for further details. 
study. Similarly, Fig. 1(b) shows a state toward the right side $(\mathrm{R})$. These figures depict systems proposed by Kelso and his colleagues and take into account the assumption in their studies to estimate the motor control of human movement as mentioned above. That is, an attractor underlies the system shown in Figs. 1(a) and 1(b), and generates the same movement pattern repeatedly and continuously. Therefore, Kelso et al. treated the small change in input as a perturbation, and examined the stability of the system " in terms of relaxation time and critical slowing down" Yamamoto \& Gohara, 2000]. On the other side, Fig. 1(c) shows the dynamics when a human switches two attractors abruptly. This mixed state shows that we are required to switch rapidly and stochastically two attractors excited with two external inputs. The dynamics of the switching state in this system implies that not only are $\mathrm{L}$ and $\mathrm{R}$ excited with the external input but also the dynamics is affected by the temporal order and the temporal interval of the two external inputs. In this system, the number of the attractors corresponds to the number of the external inputs. This phenomenon is called the switching dynamics, wherein the human has to switch several attractors Yamamoto \& Gohara, 2000]. We need to adopt the latter approach and the corresponding theory to examine this dynamics in order to study human motor control, and the difference between this study and the studies by Kelso and his colleagues is that the experiment in our study is based on the nonautonomous dynamical systems theory.

The first study in which the dynamical systems theory Gohara \& Okuyama, 1999a involving switching stochastically with several external inputs was applied to human movement was carried out by Yamamoto and Gohara [2000]. They focused on the periodic movement patterns of forehand and backhand strokes of tennis players as attractors were excited with external inputs. They confirmed that a fractal-like structure exists in the transition state between the two movement patterns when players switch patterns stochastically and rapidly. They also provided evidence that the fractal-like geometric structure is a Cantor set. Suzuki and Yamamoto 2015] examined the relationship between transition states, which occurred by excited attractors, and skill levels for table tennis by the switching-time length $T$ as a parameter of the external input. The theoretical background is that the transition states are influenced by $T$, and the authors quantified these states by using a fractal dimension, i.e. the value of the correlation dimension. As a result, the values of the correlation dimension, which are an indicator of the complexity of behavior in movement patterns, for unskilled players when $T$ is longer are the same as values for skilled players when $T$ is shorter. This study suggested that $T$ and the state of complexity, i.e. the correlation dimension, are closely related to each other. Furthermore, a regular relationship exists between $T$ and the fractal dimension Hirakawa et al., 2016].

Previous studies Nishikawa \& Gohara, 2008; Sato \& Gohara, 2001: Wada \& Gohara, 2001a, 2001b] in applied physics have examined this kind of regular relationship in various physical phenomena. Until now, few previous studies have been conducted in which human movement has been analyzed Hirakawa et al., 2016]; hence, accumulating examinations of various human movements within the context of nonautonomous dynamical systems theory is essential. For example, Hirakawa et al. [2016] analyzed continuous trajectories of a fingertip in pointing movements toward left and right directions. However, such a task might be considered a discrete task, because it involves a reaching movement toward two preset locations. Therefore, in this study we investigate continuously moving left or right target locations as the external inputs in the task. This entails developing a task in which participants must switch a fingertip movement toward the left and right directions while they track the target continuously. The purpose of this study is to estimate the transition state between attractors that are excited by $T$, which is the external parameter, and to examine the regular relationship between $T$ and the transition state when participants track a moving target continuously as the external input. Furthermore, based on these results, we will be able to demonstrate that the dissipative dynamical system, which is applied for human movement, is stochastically excited by plural temporal inputs characterized by the trajectory set $\Gamma(C)$ starting from the initial set $C$. We also show the relationship between Eq. (2) as the discrete dynamical system and Eq. (3) as the continuous dynamical system from the perspective of the nonautonomous dynamical system by analyzing the set on the Poincaré section $\Sigma$ in the hyper-cylindrical state space $\mathcal{M}$. 


\section{Dynamical System Excited by Switching Inputs}

From the discussion in the preceding section, it seems not appropriate to study human motor control with only the perspective of autonomous dynamical system that is incapable of addressing the switching between several movement patterns following an abrupt change in the temporal input Yamamoto \& Gohara, 2000]. Hence, we perform the experiment in this study with the dissipative nonautonomous dynamical system theory Gohara \& Okuyama, 1999b; Nishikawa \& Gohara, 2008]. We briefly survey this theory in this section.

An autonomous system is expressed as $\dot{x}=$ $f(x)$. A nonautonomous system depending on a continuous external input $I(t)$ is defined by the following ordinary differential equation:

$$
\dot{x}=f[x, I(t)], \quad x, I \in R^{N},
$$

where $x$ and $f$ are the state and the vector fields, respectively. In the nonautonomous system, we consider the manner in which the vector field changes with the external input $I(t)$ over time. The input is the set $\{I\}_{l=1}^{L}$ under a finite time length, and is stochastically and continuously fed into the system. The attractor is excited by $I(t)$ in this system. We assume several attractors in a nonautonomous system, and we examine the transition states between attractors. The trajectories in the state space are unable to converge sufficiently onto those attractors if the external input $I(t)$ is switched rapidly and stochastically. If a sequence of the input is repeated randomly, the set $C$ on the Poincaré section $\Sigma$ satisfies the following equation:

$$
C=\bigcup_{l=1}^{L} g_{l}(C)
$$

and the set $C$ consists of $g_{l}(C)$. Moreover, this set $C$ influences the trajectory in the hyper-cylindrical state space $\mathcal{M}$. The set of trajectories $\Gamma(C)$ starting from the initial set $C$ in state space $\mathcal{M}$ is the bundle of trajectories $\gamma_{l}(C)$ that was influenced by each external input $I_{l}$ :

$$
\Gamma(C)=\bigcup_{l=1}^{L} \gamma_{l}(C) .
$$

We are able to quantify the set $C$ in this state space as the value of the correlation dimension calculated by the typical method to estimate a feature of a fractal state Strogatz, 2001]. Furthermore, we are able to estimate the relationship between the set $C$ or the set of trajectories $\Gamma(C)$ and the switchingtime length $T$ Gohara \& Okuvama, 1999a. $T$ is also the duration of the external input. That is, although the set on the Poincaré section $\Sigma$ is of discrete points, the set on the Poincaré section $\Sigma$ is originally one trajectory, and the connection between the discrete dynamical system [Eq. (2)] and the continuous dynamical system [Eq. (3)] is on the Poincaré section $\Sigma$. Therefore, it is valid to apply the nonautonomous dynamical system to an experiment designed to investigate human movement patterns switched continuously and abruptly. We omit the detailed theoretical explanation of this dynamical system in which the input pattern is stochastically changed such as for a nonautonomous svstem. and refer the reader to [Gohara \& Okuyama, 1999a].

\section{Methods}

\subsection{Participants}

The seven participants were healthy right-handed men (according to the laterality questionnaire on the Edinburgh Handedness Inventory Oldfield, 1971]) and had no vision problems. Their ages ranged between 21 and 39 years (mean $M=32.6$ and standard deviation $S D=4.2$ ). They had no history of neurological or musculoskeletal disorders. All participants provided written informed consent after the experimenter explained the experimental procedure. The procedure used in this study was approved by the Human Ethics Committee of Nanzan University.

\subsection{Experimental setting}

Figure 2 shows the experimental setup. Eight lightemitting diodes (LEDs), each with a diameter $\phi=$ $5 \mathrm{~mm}$, were located in a straight line at the left and right positions and at the back of the slider, respectively. The distance between two LEDs was $5 \mathrm{~mm}$. The distance between the sets of eight LEDs at the left and right sides was $170 \mathrm{~mm}$. A lever, used by a participant to track the LEDs lit, was set on the slider and participants could move the lever freely toward left and right directions. The slider was set on a table. Each participant sat $300 \mathrm{~mm}$ in front of the slider and could adjust the height of the chair. The movement of a reflective marker 


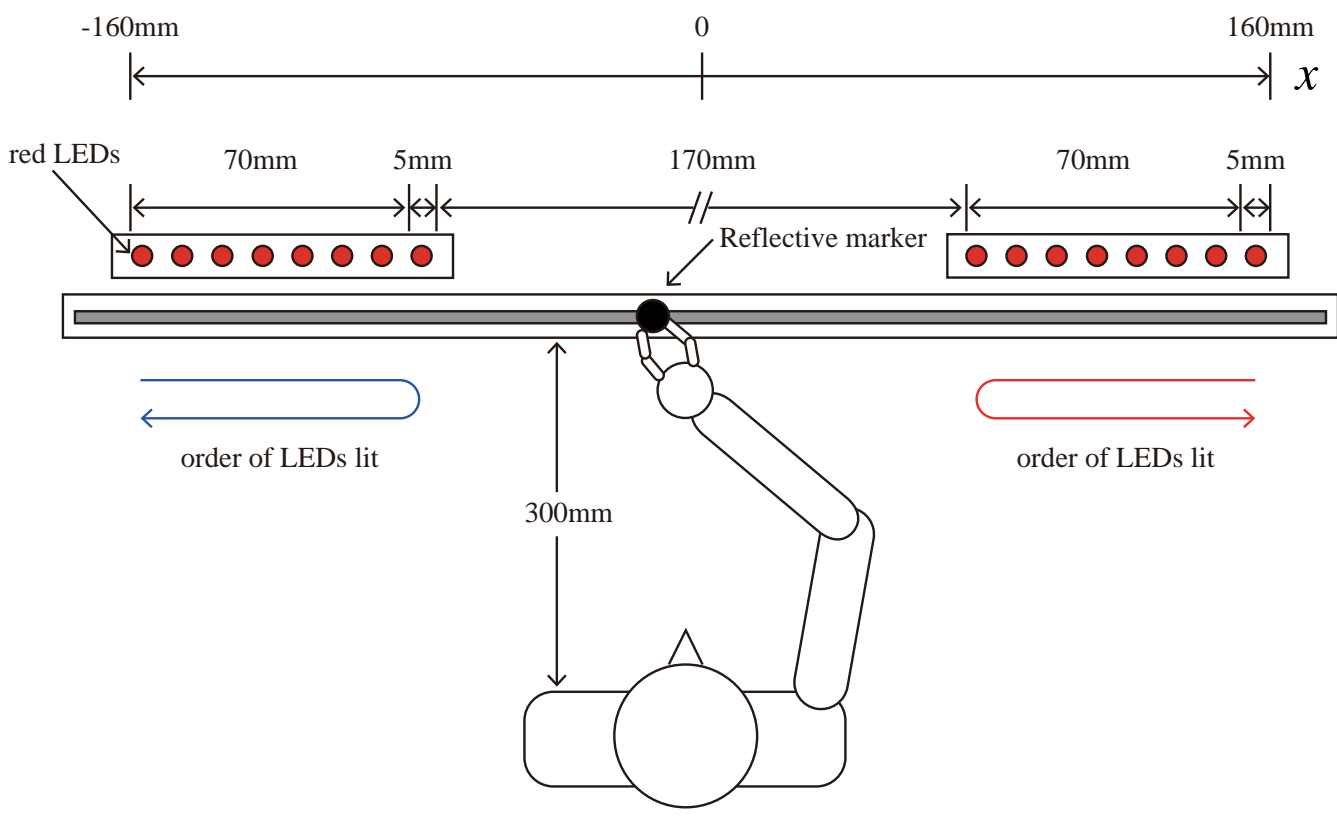

Fig. 2. Experimental setup.

(15 mm in diameter) pasted onto the lever was sampled at $240 \mathrm{~Hz}$ by an optical motion-tracking system (Qualisys Oqus 300, Gothenburg, Sweden). The lighting of the LEDs was controlled with LabVIEW programming (Version 8.6, National Instruments, Austin TX; NI cDAQ-9174, NI9403), and was synchronized with Oqus.

\subsection{Task and procedure}

The experiment was conducted in a quiet room. Participants first held the lever with thumb and index finger. They were then instructed to track the LEDs lit. The order of LED lighting was as follows. When the LEDs located to the left of the participant (L) were lit, the lighting of LEDs moved to the right one by one after the LED on the leftmost was first lit. The lighting of LEDs moved to the left one by one after the rightmost LED was lit. When the LEDs located to the right of the participant $(\mathrm{R})$ were lit, the order of LEDs lit was the reverse of that of $\mathrm{L}$. The durations of LEDs lit on $\mathrm{L}$ or R, i.e. the switching-time lengths $T$ s were 2.00 , $1.00,0.75,0.50,0.35$, and $0.25 \mathrm{~s}$. The experiment was divided into two or three days, and measurements were performed under up to three switchingtime lengths per day.

Participants executed the periodic-input condition in which only L (LLL...) or only R (RRR...) was repeated as well as the switching-input condition in which $\mathrm{L}$ and $\mathrm{R}$ were switched stochastically and randomly for each $T$. For each $T$, L or $\mathrm{R}$ was repeated for 62 trials during the periodic-input condition and for 496 trials during the switchinginput condition. The number of trials under the switching-input condition was divided into eight blocks to equalize the number per block under the periodic-input condition, i.e. 62 trials per block. Participants performed ten blocks, i.e. two blocks under the periodic-input condition and eight blocks under the switching-input condition, for each $T$. For the switching-input condition, the input sequence consisted of only eight patterns, i.e. LLL, RLL, LRL, RRL, LLR, RLR, LRR, and RRR, although the sequence was made up stochastically and randomly. The first two trials for each block after the second block were the same as the last two trials for the previous block. The first two trials after the second block were removed for the data analysis. The data analyzed consisted of 60 trials for the periodic-input condition and 480 trials $(=8$ blocks $\times(62-2)$ trials $=8$ serial patterns $\times 60$ data) for the switching-input condition. We set up the number of participants and data set by applying the previous studies Hirakawa et al., 2016; Suzuki \& Yamamoto, 2015] with dynamical systems theory for human movement. The number and duration of a break between blocks was determined freely by each participant according to his need. 


\subsection{Data analysis}

The displacement of the position of the lever was calculated using Qualisys Track Manager (Gothenburg, Sweden). The displacement data were smoothed with a second-order Butterworth digital low-pass filter using a $5-\mathrm{Hz}$ cutoff frequency to obtain the positional data $x$. The velocity $\dot{x}$ was calculated by differentiating $x$. We embedded $x$ and $\dot{x}$ into the hyper-cylindrical state space $\mathcal{M}$ to examine the trajectories in a continuous dynamical system. The Poincaré section $\Sigma(\theta=0)$ in state space $\mathcal{M}$ is

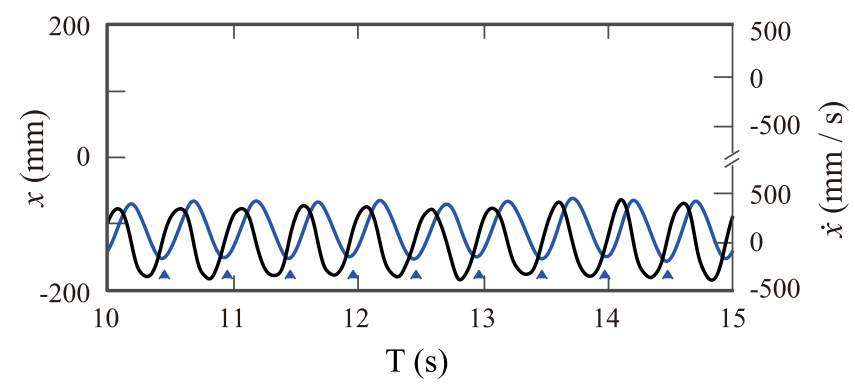

(a)

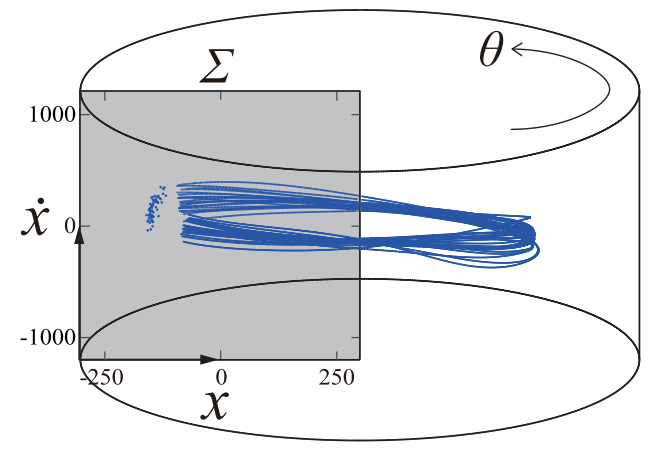

(c)

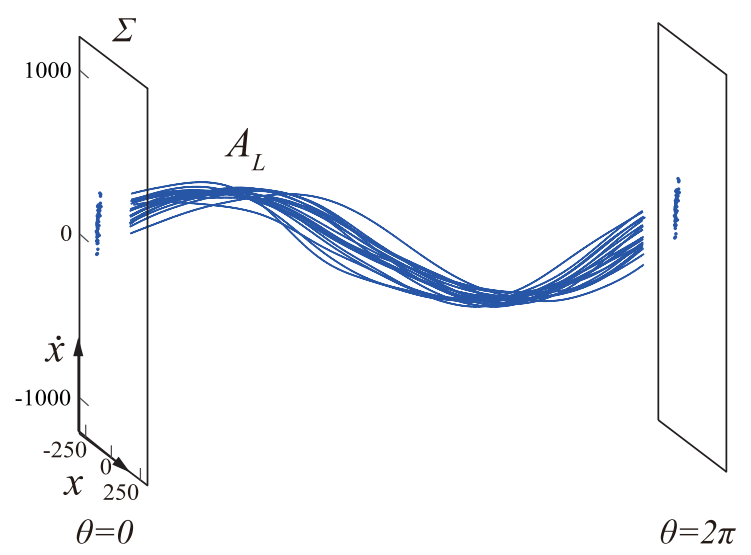

(e) the moment when the leftmost LED was lit (when L started) and the moment when the rightmost LED was lit (when $\mathrm{R}$ started), as denoted by the blue and red triangles in Figs. 3(a), 3(b), and 5(a). We unfolded state space $\mathcal{M}$ to $\theta-(x, \dot{x})$ space to examine the relationship between the trajectory sets $\Gamma(C)$ and $g(C)$. The correlation dimension $D_{2}$ for set $g(C)$ on the Poincaré section $\Sigma(\theta=2 \pi)$ under each $T$ was calculated with the G-P algorithm [Grassberger \& Procaccia, 1983] by using the correlation integral, which is a typical method to estimate the

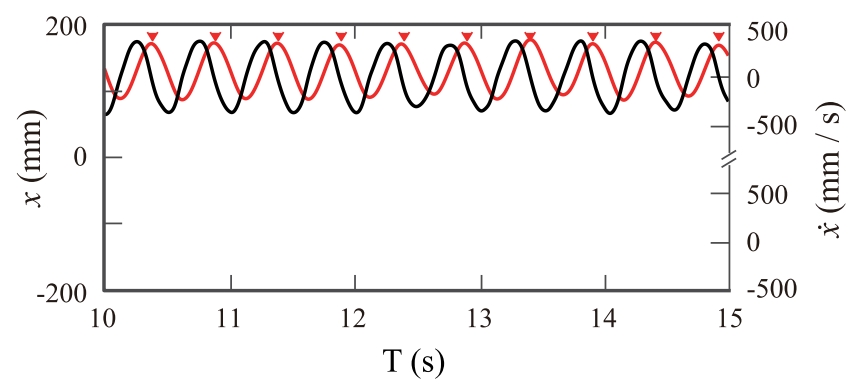

(b)

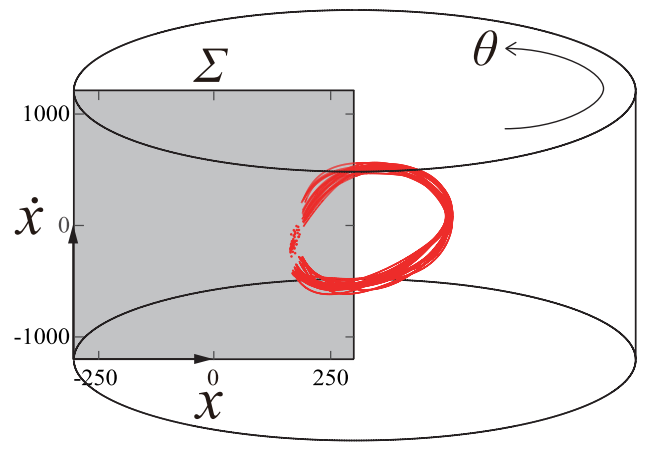

(d)

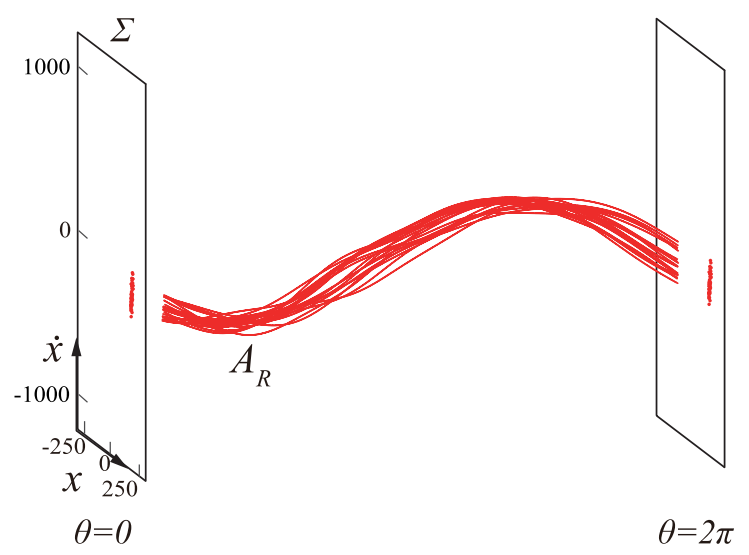

(f)

Fig. 3. Dynamics for the periodic-input condition (using data from participant \# 7 for a switching-time length $T$ of $0.25 \mathrm{~s}$ ). 
fractal dimension. The validity and reliability of a fitting curve were evaluated by using Akaike's information criterion (AIC) to estimate the relationship between $T$ and $D_{2}$.

\section{Results}

\subsection{Periodic-input condition}

Figure [3(a) shows part of the time series data of the output pattern $(5 \mathrm{~s})$ for participant \#7 to track L continuously under the periodic-input condition when the switching-time length $T$ (duration of $\mathrm{L}$ lit) is $0.25 \mathrm{~s}$ as a typical example. The reason why we selected data for participant \#7 is that this result in Fig. 9, which is described later, was the most approximate to the fitting curve; i.e. this value is the minimum by the least-squares method. Similarly, Fig. 3(b) shows part of the result for participant $\# 7$ to track $R$. The vertical scale on the left of these figures is $x$, and the scales on the right side are $\dot{x}$. The blue and red triangles indicate when $\mathrm{L}$ and $\mathrm{R}$ started to light up, respectively. The blue and red solid lines are the positional displacement $x$ of the lever when a target LED was lit on $\mathrm{L}$ or $\mathrm{R}$, respectively. The black solid lines are the velocity $\dot{x}$.
Figure 3(c) shows the result when output patterns were embedded into the hyper-cylindrical state space $\mathcal{M}$ when participant $\# 7$ tracked L. The Poincaré section $\Sigma(\theta=0,2 \pi)$ is the moment that $\mathrm{L}$ started. The trajectory around the cylinder represents one cycle and was composed of $x$ and $\dot{x}$ during the time the LEDs on $\mathrm{L}$ were lighting up. Figure 3(d) shows the result when participant \#7 tracked R. The trajectories around the cylinder represent 60 cycles. Additionally, the trajectories before and after the Poincaré section $\Sigma(\theta=0,2 \pi)$ in the state space were omitted to more easily confirm the set $C$ on the section.

Figure 3(e) shows the result when we unfolded Fig. 3(c) in $\theta-(x, \dot{x})$ space to more easily examine the set of trajectories in state space $\mathcal{M}$. Figure $3(\mathrm{f})$ shows the result corresponding to Fig. 3(d). These trajectories were the output patterns when the participant tracked $\mathrm{L}$ or $\mathrm{R}$, and the trajectories in the state space were clearly different according to the type of external input, i.e. L or R.

Figure 4 shows the results when we, respectively, embedded the trajectories set in state space $\mathcal{M}$ to confirm the output patterns during five $T \mathrm{~s}$ (except for $T=0.25 \mathrm{~s}$ ) under the periodic-input condition. Figures 4(a)-4(e) show the results for

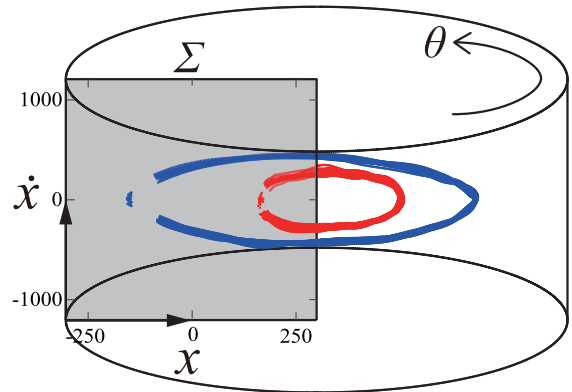

(a)

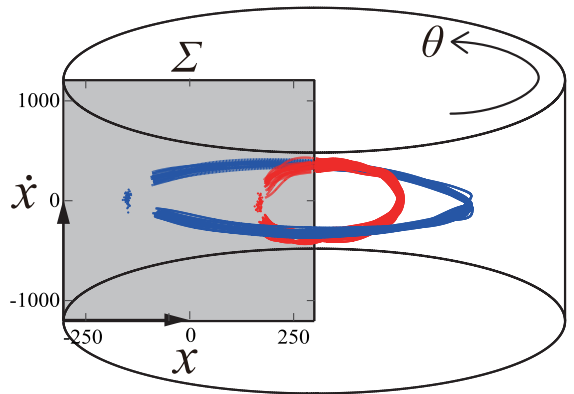

(b)

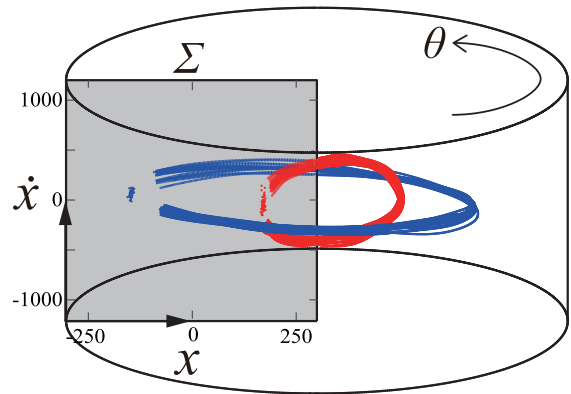

(c)

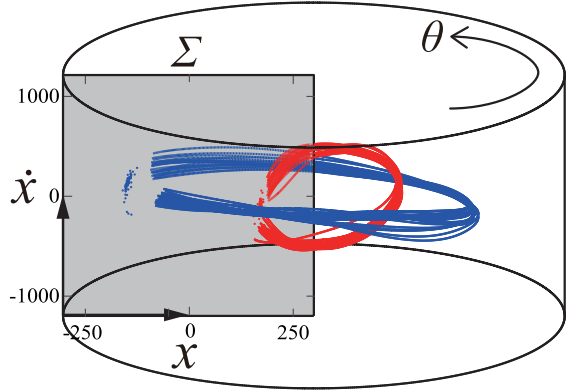

(d)

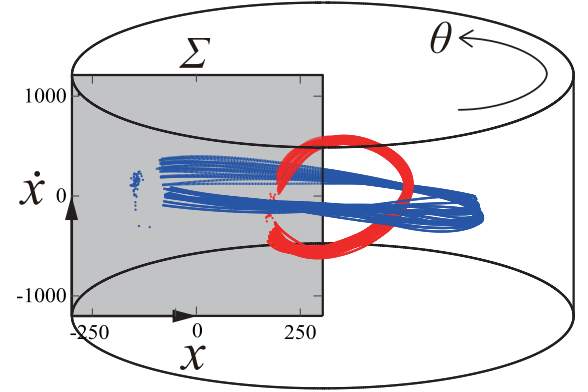

(e)

Fig. 4. Cylindrical state space $\mathcal{M}$ during the periodic-input condition (using data from participant \#7). The switching-time length $T=$ (a) 2.00, (b) 1.00, (c) 0.75 , (d) 0.50, and (e) $0.35 \mathrm{~s}$. 
$T=2.00,1.00,0.75,0.50$, and $0.35 \mathrm{~s}$, respectively. The results for $T=0.25 \mathrm{~s}$ are divided into Figs. B(c) and 3(d). The trajectory around the cylinder represents one cycle, and the trajectories start from the moment when $\mathrm{L}$ or $\mathrm{R}$ started, i.e. the Poincaré section $\Sigma(\theta=0)$, and terminate at the moment when $\mathrm{L}$ or $\mathrm{R}$ ended, i.e. $\theta=2 \pi$, similar to state space $\mathcal{M}$ in Figs. 3(c) and 3(d). The blue and red trajectories are the output patterns $(x, \dot{x})$ when a participant tracked $\mathrm{L}$ and $\mathrm{R}$, respectively. In addition, the trajectories before and after the Poincaré section $\Sigma(\theta=0,2 \pi)$ were omitted to confirm the set $C$ on the section more easily. These results show that the trajectories of output patterns, which were excited by the external inputs $\mathrm{L}$ and $\mathrm{R}$, were different in the continuous dynamical system.

Furthermore, a one-way multivariate analysis of variance (MANOVA) (Wilks' $\lambda$ ) was performed to test for the equality of the means of the multiple variables in the cluster of the set on the Poincaré section for each $T$ and for all participants to examine the output patterns statistically in the discrete dynamical system, because the set of trajectories in the continuous dynamical system were different qualitatively. Table 1 lists the results. These results revealed significant differences for all participants and all Ts $(p<0.001)$, revealing that the output patterns excited by the external inputs $\mathrm{L}$ and $\mathrm{R}$ were clearly different in state space $\mathcal{M}$.

These qualitative and quantitative results under the periodic-input condition suggest that the set of trajectories - the continuous dynamical system - in the hyper-cylindrical state space $\mathcal{M}$ and the set $C$ - the discrete dynamical system - on the Poincaré section $\Sigma(\theta=2 \pi)$ were basic movement patterns excited by the external input. These movement patterns are limit-cycle attractors, and the attractors are excited by an external input, i.e. the movement patterns have obviously different excited attractors, $A_{L}$ and $A_{R}$. However, we are unable to confirm whether the output patterns during a continuous tracking movement in the periodic-input condition are attracted to either attractor when we rapidly switch the tracking movements by the external inputs $\mathrm{L}$ and $\mathrm{R}$. We examine this phenomenon in the switching-input condition.

\subsection{Switching-input condition}

Figure 5(a) shows part of the time series data of the output pattern $(5 \mathrm{~s})$ for participant \# 7 to track the LEDs continuously under the switching-input condition when the switching-time length $T$ (duration of $\mathrm{L}$ or $\mathrm{R}$ lit) is $0.25 \mathrm{~s}$ as a typical example. The reason why we selected data for participant \#7 is similar to that stated in the previous subsection. The vertical scale on the left side in Fig. 5(a) is $x$, and the scales on the right side are $\dot{x}$. The blue and red triangles indicate when $\mathrm{L}$ and $\mathrm{R}$ started to light up, respectively. The blue and red solid lines are the positional displacements of the lever $x$ when an LED on $\mathrm{L}$ or $\mathrm{R}$ was lit, respectively. The black solid line is $\dot{x}$.

Figure 5(b) shows the result when the output patterns were embedded into the hyper-cylindrical state space $\mathcal{M}$ when participant $\# 7$ tracked an LED on L or R. The Poincaré section $\Sigma(\theta=0,2 \pi)$ is the moment when $\mathrm{L}$ or $\mathrm{R}$ started. The trajectory around the cylinder was composed of $x$ and $\dot{x}$. The set $C$ on the Poincaré section $\Sigma$ comprise all data for the participant. Only 15 trajectories $\gamma(C)$ for each serial (for example, LLL) were embedded to more easily confirm the trajectories in this state space. That is, all 120 trajectories for

Table 1. Results of a MANOVA (Wilks' $\lambda$ ) of differences between the mean left $(\mathrm{L})$ and right side $(\mathrm{R})$ input patterns on the Poincaré section $\Sigma(\theta=2 \pi)$ under the periodic-input condition.

\begin{tabular}{|c|c|c|c|c|c|c|c|}
\hline \multirow[b]{2}{*}{ Participant } & & \multicolumn{6}{|c|}{$T(\mathrm{~s})$} \\
\hline & & 2.00 & 1.00 & 0.75 & 0.50 & 0.35 & 0.25 \\
\hline$\# 1$ & $\mathrm{~L} / \mathrm{R}$ & 0.0004 & 0.0016 & 0.0006 & 0.0015 & 0.0021 & 0.0009 \\
\hline$\# 2$ & $\mathrm{~L} / \mathrm{R}$ & 0.0003 & 0.0005 & 0.0012 & 0.0011 & 0.0010 & 0.0006 \\
\hline$\# 3$ & $\mathrm{~L} / \mathrm{R}$ & 0.0001 & 0.0002 & 0.0005 & 0.0002 & 0.0005 & 0.0003 \\
\hline$\# 4$ & $\mathrm{~L} / \mathrm{R}$ & 0.0003 & 0.0006 & 0.0005 & 0.0005 & 0.0008 & 0.0009 \\
\hline$\# 5$ & $\mathrm{~L} / \mathrm{R}$ & 0.0002 & 0.0004 & 0.0004 & 0.0021 & 0.0049 & 0.0035 \\
\hline$\# 6$ & $\mathrm{~L} / \mathrm{R}$ & 0.0006 & 0.0006 & 0.0010 & 0.0013 & 0.0017 & 0.0013 \\
\hline$\# 7$ & $\mathrm{~L} / \mathrm{R}$ & 0.0003 & 0.0005 & 0.0003 & 0.0007 & 0.0011 & 0.0011 \\
\hline
\end{tabular}

$p<0.001$ 


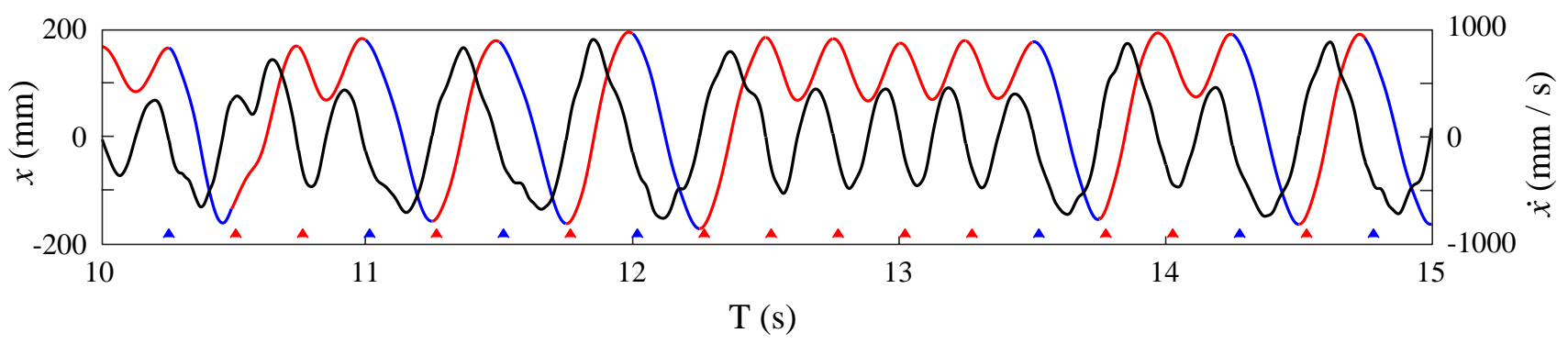

(a)

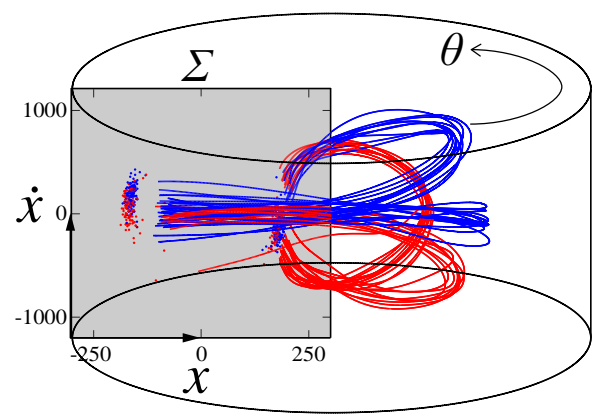

(b)

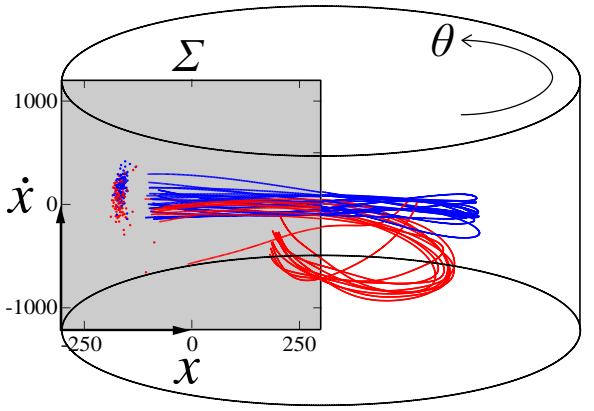

(c)

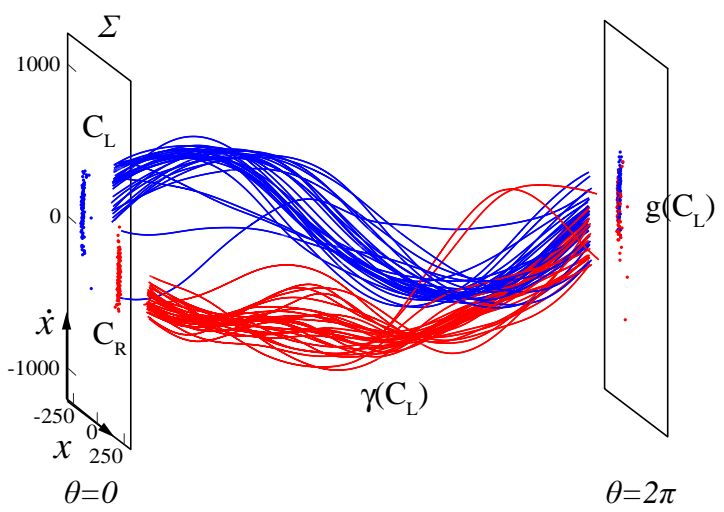

(e)

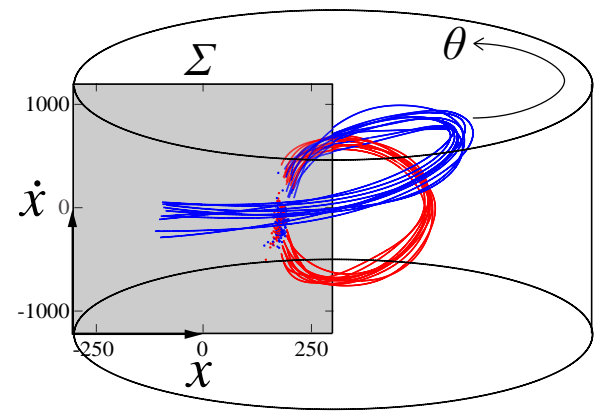

(d)

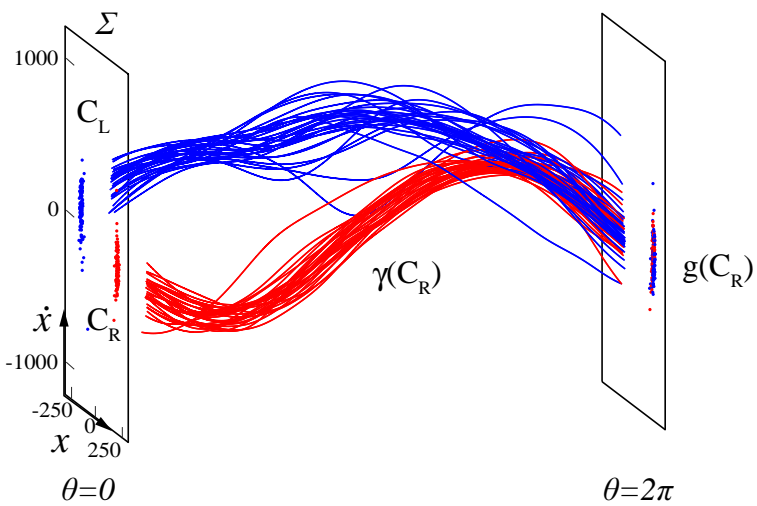

(f)

Fig. 5. Dynamics for the switching-input condition (using data from participant $\# 7$ for a switching-time length $T$ of $0.25 \mathrm{~s}$ ). 
eight serials (LLL, RLL, LRL, RRL, LLR, RLR, LRR, and RRR) were embedded in this state space. Additionally, the trajectories before and after the Poincaré section $\Sigma(\theta=0,2 \pi)$ in this state space were omitted to more easily confirm the set $C$ on the section. Figure 5(c) shows the output patterns under the switching-input condition when the current external input was L. Figure 5(d) shows the result when the current external input was $\mathrm{R}$. In these figures, the blue trajectories are the result when the preceding input was $\mathrm{L}$, and red trajectories indicate the result when the preceding input was R. That is, LL, RL, LR, and RR, which were composed of the preceding and current inputs, are represented as blue, red, blue, and red colors, respectively.

Figure 5(e) shows the result when we unfolded Fig. 5 (c) in $\theta-(x, \dot{x})$ space to more easily examine the set of trajectories in state space $\mathcal{M}$. Figure 5 (f) shows the result corresponding to Fig. 5(d). Further, the trajectories before and after the Poincaré section $\Sigma(\theta=0,2 \pi)$ in the state space were omitted to more easily confirm the set $C$ on the section. This result indicates that, under the switching-input condition, the sets of trajectories $\gamma\left(C_{L}\right)$ and $\gamma\left(C_{R}\right)$ were output patterns when the participant was tracking $\mathrm{L}$ and $\mathrm{R}$, respectively, and that the trajectories were starting from the initial sets $C_{L}$ and $C_{R}$ at $\theta=0$ and were terminated to sets $g\left(C_{L}\right)$ and $g\left(C_{R}\right)$ at $\theta=2 \pi$. For example, we are able to confirm that two clusters of the set of trajectories started from two different clusters of the initial set $C$ on the section $\Sigma(\theta=0)$ in both Figs. $5(\mathrm{e})$ and $5(f)$. These clusters show that the current output pattern was influenced by not only the current input pattern but also the preceding input pattern. That is, trajectories $\gamma\left(C_{L}\right)$ start from two clusters when the preceding input is $\mathrm{L}$ or $\mathrm{R}$ and the current input is L. Therefore, these clusters are composed of serials of external inputs LL and RL. Similarly, trajectories $\gamma\left(C_{R}\right)$ start from two clusters when the preceding input is $\mathrm{L}$ or $\mathrm{R}$ and the current input is $\mathrm{R}$. Therefore, these clusters are composed of serials of external inputs LR and RR. Based on these results, the trajectories of the continuous tracking movement in state space $\mathcal{M}$ and space $\theta-(x, \dot{x})$ were composed of four clusters with the external input.

Figure [6] shows the result when we, respectively, embedded the set of trajectories in state space $\mathcal{M}$ to confirm the output patterns during five $T$ s (except for $T=0.25 \mathrm{~s}$ ) under the switching-input condition. Figures 6(a) 6(e) show the results for $T=2.00$,

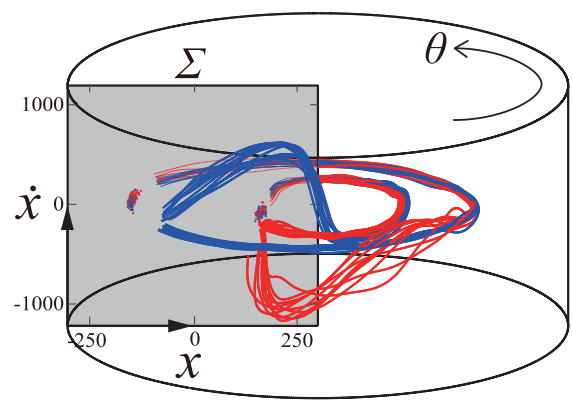

(a)

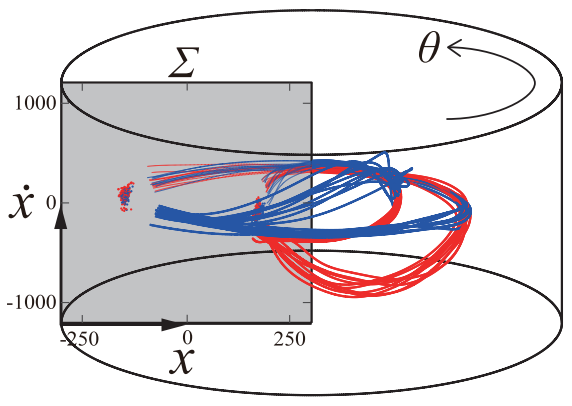

(b)

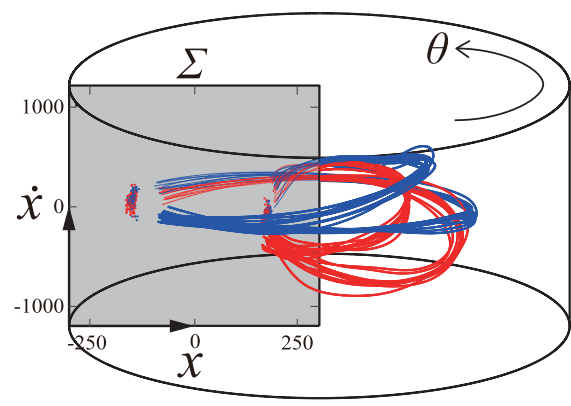

(c)

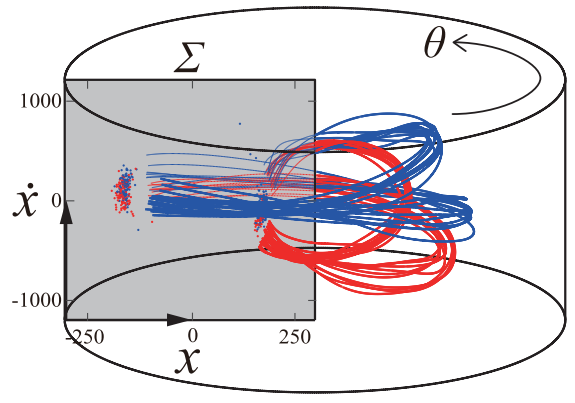

(d)

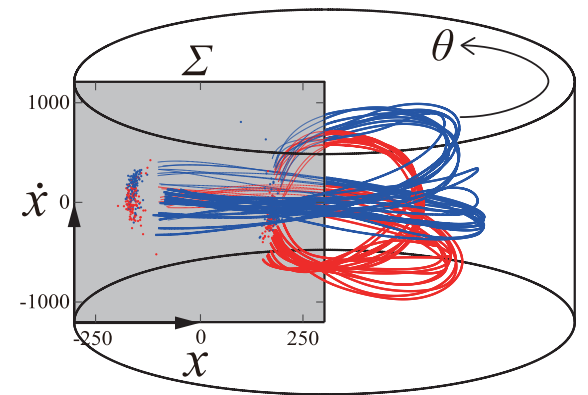

(e)

Fig. 6. Cylindrical state space $\mathcal{M}$ during the switching-input condition (using data from participant \#7). The switching-time length $T=$ (a) 2.00, (b) 1.00, (c) 0.75, (d) 0.50, and (e) $0.35 \mathrm{~s}$. 
$1.00,0.75,0.50$, and $0.35 \mathrm{~s}$, respectively. The results for $T=0.25 \mathrm{~s}$ are shown in Fig. 5 (b). The trajectory around the cylinder represents one cycle, and the trajectories start from the moment when L or $\mathrm{R}$ started, i.e. the Poincaré section $\Sigma(\theta=0)$, and terminate at the moment when $\mathrm{L}$ or $\mathrm{R}$ ended, i.e. $\theta=2 \pi$, similar to Figs. 5 (b) 5 (d). The blue and red trajectories are the output patterns $(x, \dot{x})$, and the colors are similar to that in Figs. 5 (b) - 5 (d), respectively. Additionally, the trajectories before and after the Poincaré section $\Sigma(\theta=0,2 \pi)$ were omitted to more easily confirm the set $C$ on the section. Compared with the set of trajectories under the periodicinput condition in Figs. 3 and 4 , the set of trajectories under the switching-input condition in Figs. 5 and $[6$ was excited by the external inputs $\mathrm{L}$ and $\mathrm{R}$, i.e. $\gamma\left(C_{L}\right)$ and $\gamma\left(C_{R}\right)$, which were clearly different in the continuous dynamical system.

Furthermore, a MANOVA (Wilks' $\lambda$ ) was performed to test for the equality of the means of the multiple variables in the set on the Poincare section (LL/RL and LR/RR, which are the serials composed of the preceding and the current external inputs, respectively) for each $T$ and all participants to examine the output patterns statistically in a discrete dynamical system, because the sets of trajectories in the continuous dynamical system were different qualitatively. Table 2 lists the results. Figure 7 shows a scatter diagram on the section $\Sigma$; participants \#1 to \#7 are arranged by row and the six $T_{\mathrm{s}}$ are arranged by column. The blue and red data points in Fig. 7 indicate that the preceding inputs are $\mathrm{L}$ and $\mathrm{R}$, respectively. The set on the left side of the section is $g\left(C_{L}\right)$, and the other is $g\left(C_{R}\right)$. The results revealed significant differences in 78 of the 84 cases $(\approx 92.6 \%)$ from the 42 sections. These results suggest that tracking movements became four clusters by second-order sequence effects composed of the preceding and current inputs, i.e. LL, RL, LR, or RR.

Figure 8 shows the result when the set of trajectories in state space $\mathcal{M}$ in Figs. 5(b) and 6 was unfolded into space $\theta-(x, \dot{x})$ to examine the clusters in more detail that were outputted by serials of external inputs. Figures $8(a)-8(f)$ show the results for $T=2.00,1.00,0.75,0.50,0.35$, and $0.25 \mathrm{~s}$, respectively. The set on the Poincaré section comprises all data (480 data points for each section), and the trajectories $\Gamma(C)$ are the averaged trajectories for every eight serials (LLL, RLL, LRL, RRL, LLR, RLR, LRR, and RRR). For example, when the input before the preceding input is $\mathrm{L}$, the preceding input is $\mathrm{L}$, and the current input is $\mathrm{L}$, then the serial is LLL. Additionally, the trajectories after each Poincaré section $\Sigma(\theta=0, \pi / 2, \pi, 3 \pi / 2)$ were omitted to more easily confirm the set $C$ on the section. The blue trajectories are for when the preceding input is $\mathrm{L}$ and the current inputs are

Table 2. Results of a MANOVA (Wilks' $\lambda$ ) of differences between the mean left side-left side (LL) and right side-left side $(\mathrm{RL})$ and between the mean right side-right side (RR) and left side-right side (LR) input patterns on the Poincaré section $\Sigma(\theta=2 \pi)$ under the switching-input condition.

\begin{tabular}{|c|c|c|c|c|c|c|c|}
\hline \multirow[b]{2}{*}{ Participant } & & \multicolumn{6}{|c|}{$T(\mathrm{~s})$} \\
\hline & & 2.00 & 1.00 & 0.75 & 0.50 & 0.35 & 0.25 \\
\hline$\# 1$ & $\begin{array}{l}\mathrm{LL} / \mathrm{RL} \\
\mathrm{LR} / \mathrm{RR}\end{array}$ & $\begin{array}{l}0.6248^{* * *} \\
0.6057^{* * *}\end{array}$ & $\begin{array}{l}0.9591^{* *} \\
0.9614^{* *}\end{array}$ & $\begin{array}{l}0.9157^{* * *} \\
0.6100^{* * *}\end{array}$ & $\begin{array}{l}0.6714^{* * *} \\
0.9161^{* * *}\end{array}$ & $\begin{array}{l}0.9275^{* * *} \\
0.9881\end{array}$ & $\begin{array}{l}0.9488^{* *} \\
0.8388^{* * *}\end{array}$ \\
\hline$\# 2$ & $\begin{array}{l}\mathrm{LL} / \mathrm{RL} \\
\mathrm{LR} / \mathrm{RR}\end{array}$ & $\begin{array}{l}0.9770 \\
0.9684^{*}\end{array}$ & $\begin{array}{l}0.9669^{*} \\
0.9860\end{array}$ & $\begin{array}{l}0.9266^{* * *} \\
0.9901\end{array}$ & $\begin{array}{l}0.9435^{* *} \\
0.8607^{* * *}\end{array}$ & $\begin{array}{l}0.7281^{* * *} \\
0.7950^{* * *}\end{array}$ & $\begin{array}{l}0.8106^{* * *} \\
0.8182^{* * *}\end{array}$ \\
\hline$\# 3$ & $\begin{array}{l}\mathrm{LL} / \mathrm{RL} \\
\mathrm{LR} / \mathrm{RR}\end{array}$ & $\begin{array}{l}0.9996 \\
0.9032^{* * *}\end{array}$ & $\begin{array}{l}0.7162^{* * *} \\
0.9693^{*}\end{array}$ & $\begin{array}{l}0.7421^{* * *} \\
0.9363^{* *}\end{array}$ & $\begin{array}{l}0.4999^{* * *} \\
0.9088^{* * *}\end{array}$ & $\begin{array}{l}0.6611^{* * *} \\
0.8418^{* * *}\end{array}$ & $\begin{array}{l}0.6246^{* * *} \\
0.8593^{* * *}\end{array}$ \\
\hline$\# 4$ & $\begin{array}{l}\mathrm{LL} / \mathrm{RL} \\
\mathrm{LR} / \mathrm{RR}\end{array}$ & $\begin{array}{l}0.9854 \\
0.7979 * * *\end{array}$ & $\begin{array}{l}0.9662^{*} \\
0.8177^{* * *}\end{array}$ & $\begin{array}{l}0.8779^{* * *} \\
0.8869^{* * *}\end{array}$ & $\begin{array}{l}0.8989^{* * *} \\
0.8250^{* * *}\end{array}$ & $\begin{array}{l}0.9462^{* *} \\
0.9537^{* *}\end{array}$ & $\begin{array}{l}0.6288^{* * *} \\
0.7886^{* * *}\end{array}$ \\
\hline$\# 5$ & $\begin{array}{l}\mathrm{LL} / \mathrm{RL} \\
\mathrm{LR} / \mathrm{RR}\end{array}$ & $\begin{array}{l}0.7868^{* * *} \\
0.6701^{* * *}\end{array}$ & $\begin{array}{l}0.5148^{* * *} \\
0.6936^{* * *}\end{array}$ & $\begin{array}{l}0.7914^{* * *} \\
0.9478^{* *}\end{array}$ & $\begin{array}{l}0.5120^{* * *} \\
0.6862^{* * *}\end{array}$ & $\begin{array}{l}0.5234^{* * *} \\
0.5997^{* * *}\end{array}$ & $\begin{array}{l}0.6381^{* * *} \\
0.8056^{* * *}\end{array}$ \\
\hline$\# 6$ & $\begin{array}{l}\mathrm{LL} / \mathrm{RL} \\
\mathrm{LR} / \mathrm{RR}\end{array}$ & $\begin{array}{l}0.8568^{* * *} \\
0.9701^{*}\end{array}$ & $\begin{array}{l}0.7562^{* * *} \\
0.9578^{* *}\end{array}$ & $\begin{array}{l}0.9460^{* *} \\
0.9748^{*}\end{array}$ & $\begin{array}{l}0.7467^{* * *} \\
0.9077^{* * *}\end{array}$ & $\begin{array}{l}0.6439 * * * \\
0.7170^{* * *}\end{array}$ & $\begin{array}{l}0.6335^{* * *} \\
0.8502^{* * *}\end{array}$ \\
\hline$\# 7$ & $\begin{array}{l}\mathrm{LL} / \mathrm{RL} \\
\mathrm{LR} / \mathrm{RR}\end{array}$ & $\begin{array}{l}0.9303^{* * *} \\
0.9325^{* * *}\end{array}$ & $\begin{array}{l}0.9593^{* *} \\
0.8097^{* * *}\end{array}$ & $\begin{array}{l}0.9063^{* * *} \\
0.7960^{* * *}\end{array}$ & $\begin{array}{l}0.8350^{* * *} \\
0.8468^{* * *}\end{array}$ & $\begin{array}{l}0.7397^{* * *} \\
0.9438^{* *}\end{array}$ & $\begin{array}{l}0.8039^{* * *} \\
0.9038^{* * *}\end{array}$ \\
\hline
\end{tabular}

***: $p<0.001, * *: p<0.01, *: p<0.05$. 
T. Hirakawa et al.
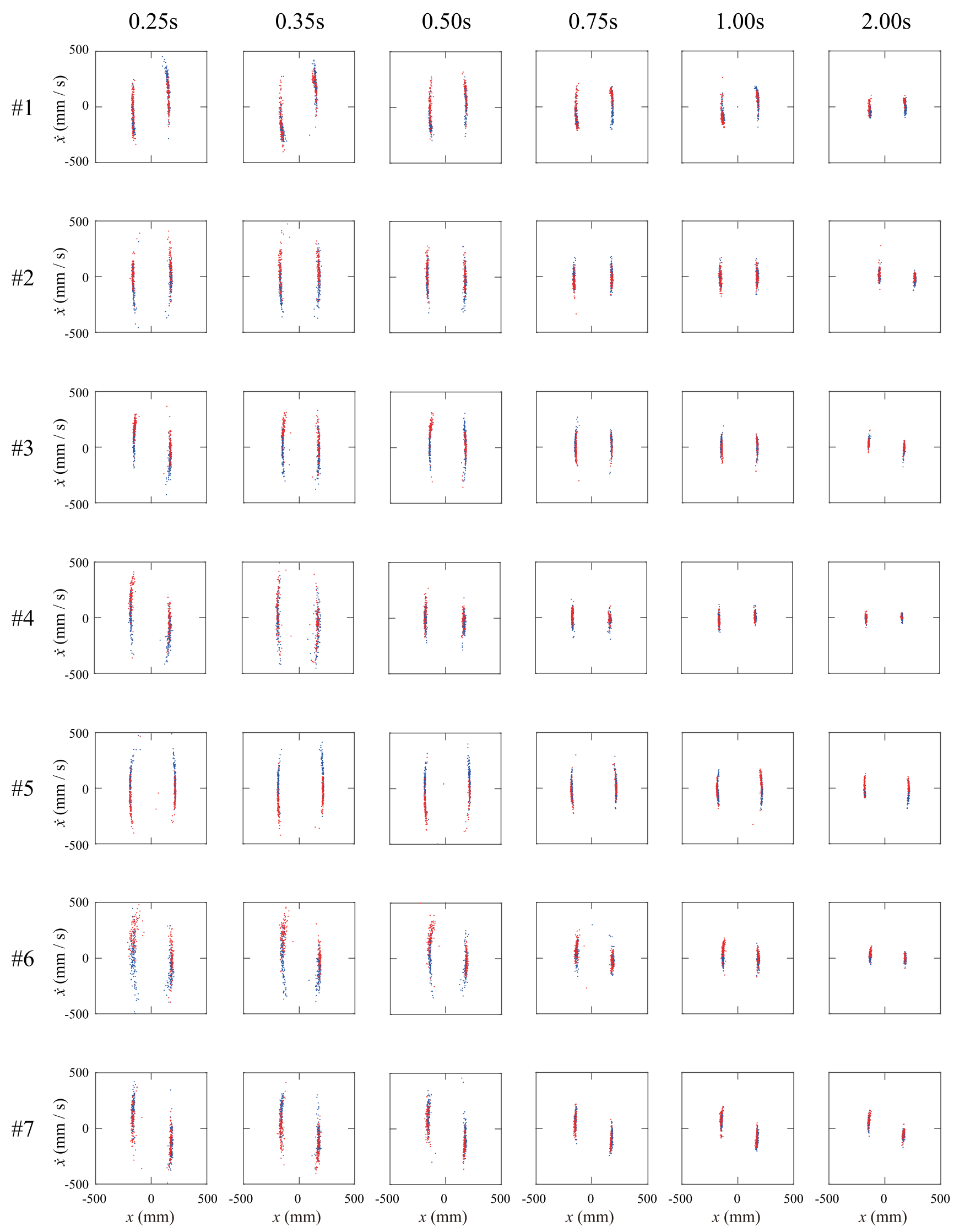

Fig. 7. Results of $g(C)$ on the Poincaré section $\Sigma(\theta=2 \pi)$. Participants $\# 1$ to \#7 are arranged by row and the six $T$ s are arranged by column. 


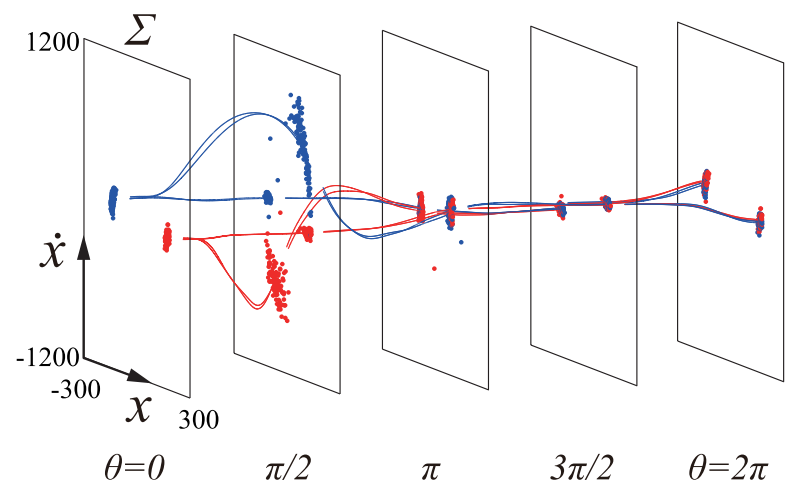

(a)

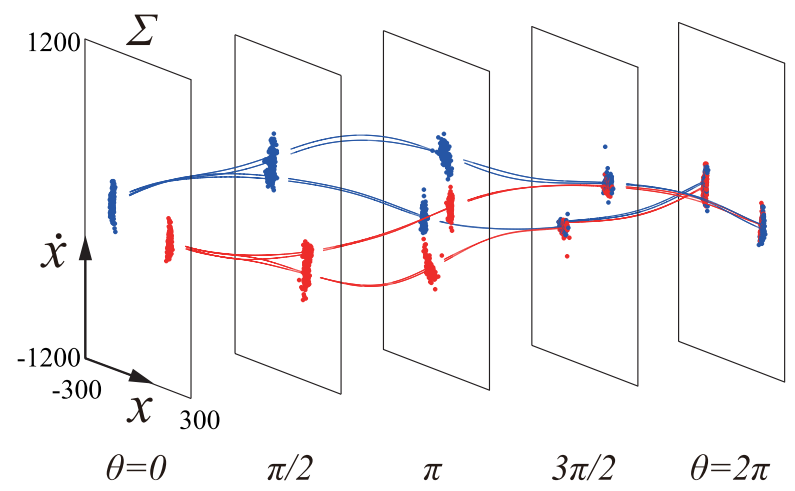

(c)

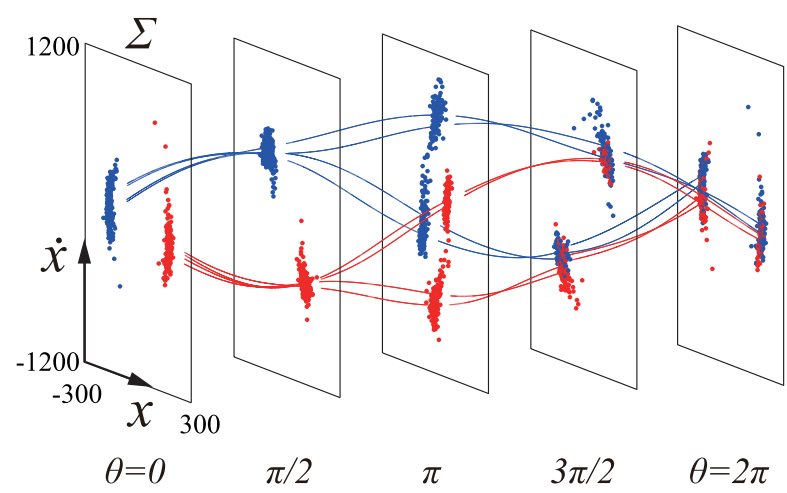

(e)

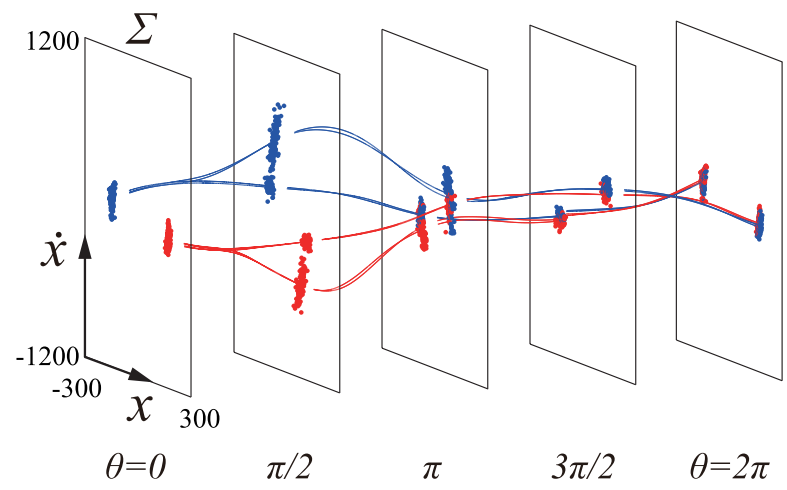

(b)

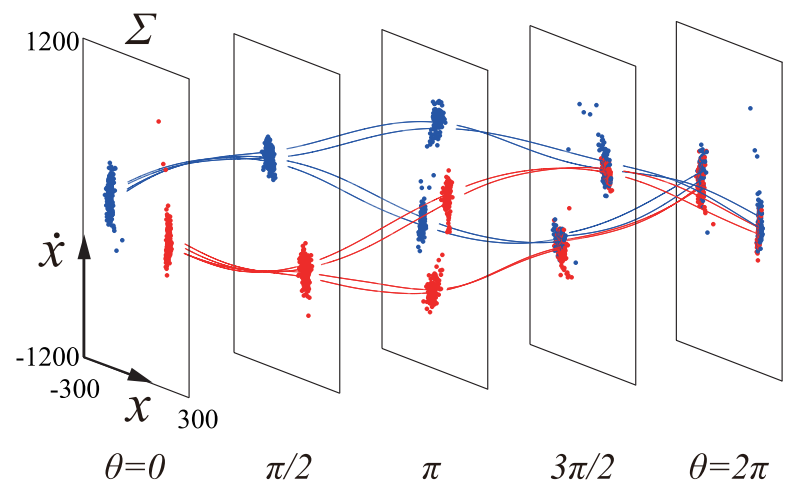

(d)

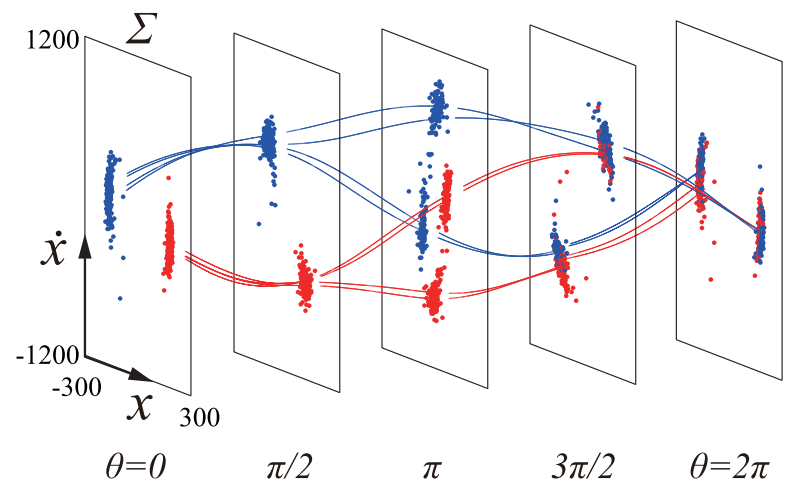

(f)

Fig. 8. The unfolded state space during the switching-input condition (using data from participant \#7). The switching-time length $T=$ (a) 2.00, (b) 1.00, (c) 0.75, (d) 0.50, (e) 0.35, and (f) $0.25 \mathrm{~s}$.

L (LL) and R (LR). Red trajectories are for when the preceding input is $\mathrm{R}$ and the current inputs are $L(R L)$ and $R(R R)$. From these results, we are able to confirm clearly the eight trajectories as $T$ shortened [for example, Figs. 8(e) and 8(f)], while the trajectories during longer $T$ s [for example, Figs. 8(a) and 8(b)] seem to converge at two points. This result suggests that we are able to confirm that the current output pattern is influenced by the preceding external input under the switchinginput condition and that we are also able to confirm a second-order sequence effect by trajectory $\Gamma(C)$ in the continuous dynamical system. Furthermore, these results indicate that not only are the four clusters composed of trajectories from each serial but also that each of the four clusters (LL, $\mathrm{RL}, \mathrm{LR}$, and RR) is composed of two subclusters, respectively. That is, the set of trajectories $\Gamma(C)$ 
is composed of eight clusters while the initial set $C$ has only four clusters on the Poincaré section. Therefore, the eight trajectories in state space $\mathcal{M}$ show that the human output patterns exhibit a fractal-like feature or a self-similarity structure by third-order sequence effects when humans switched rapidly between motions with two external inputs.

\subsection{Switching-time length $T$ and correlation dimension $\mathrm{D}_{2}$}

Figures 9(a) 9(f) show typical examples (from participant \#7) for set $g(C)$ on the Poincaré section $\Sigma(\theta=2 \pi)$ under the switching-input condition for all six $T$ s. Figures 9(a) - (f) correspond to the results for $T=0.25,0.35,0.50,0.75,1.00$, and $2.00 \mathrm{~s}$, respectively. From these results, we are able to confirm that set $g(C)$ spreads over the section $\Sigma$ during a shorter $T$ [for example, Fig. 9(a)] and converges to a local area during a longer $T$ [for example, Fig. 9(f)]. The blue and red data points in the upper part of Fig. $9(\mathrm{~g})$ are the results of the correlation dimension $D_{2}$ for each participant under each $T$. For all participants and $T$ s, the value of the dimension exceeds $\sim 1$ under the shorter switchingtime length $T$ [for example, Figs. 8(f) and 9(a)] and decreases to near zero under the longer $T$ [for example, Figs. 8(a) and 9(f)]. This suggests that set $C$ on the Poincaré section $\Sigma$ is spreading gradually from points to lines and from lines to a plane as $T$ is shortened. That is, the value of the correlation dimension becomes higher because of spreading around two excited attractors when switching these two attractors rapidly as $T \rightarrow 0$, while the value of the correlation dimension was lower because of the convergence to these two attractors as $T \rightarrow \infty$.

The black open circles in the upper part of Fig. 9(g) provide the means of the correlation dimension for all participants and each $T$. Repeated measures of ANOVA revealed a significant effect for $T[F(5,30)=34.59, p<0.01]$. Moreover, multiple comparisons revealed that the value of the correlation dimension for $T=2.00 \mathrm{~s}$ was lower than the value at other $T \mathrm{~s}$, the value for $T=1.00 \mathrm{~s}$ was lower

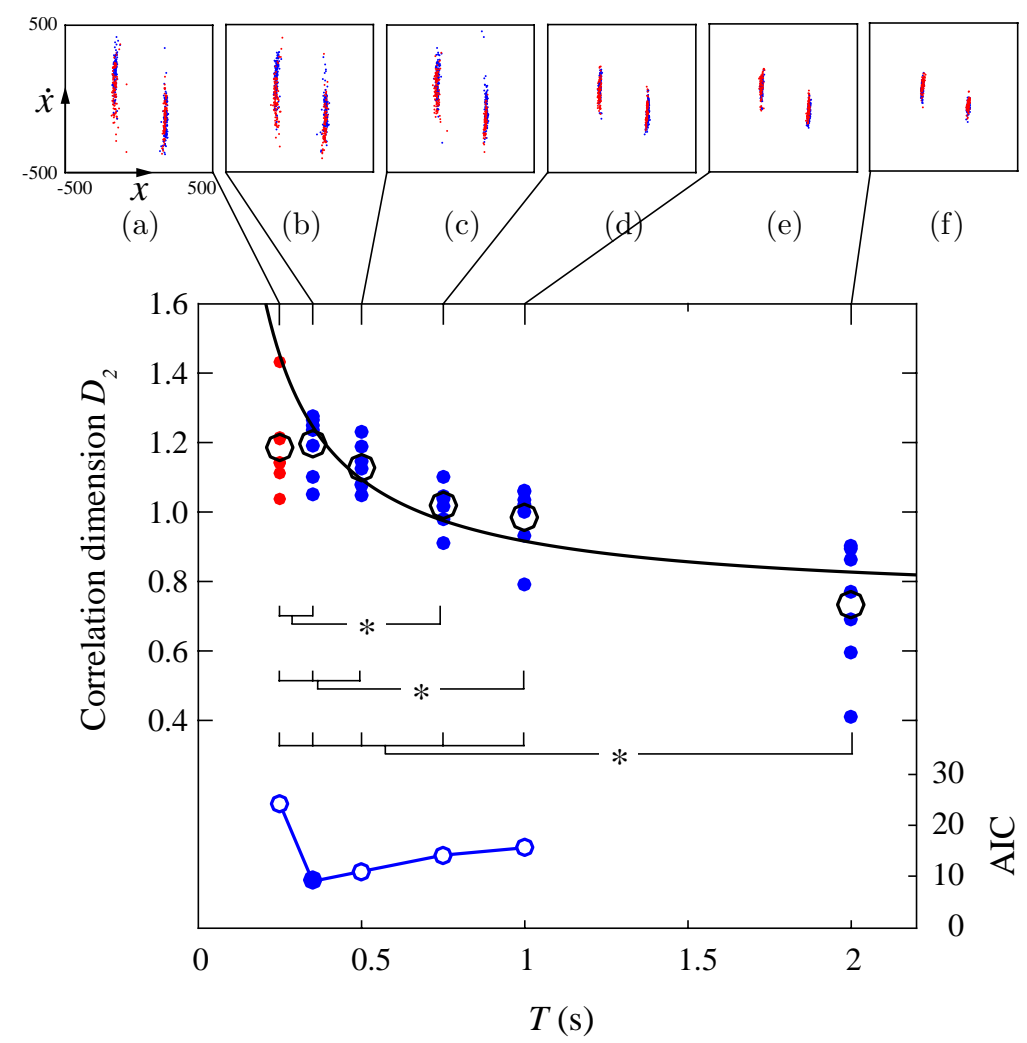

(g)

Fig. 9. Means of the correlation dimensions and the raw data under all switching-time lengths and the fitting curve, results of ANOVA, and AIC. The top panels are examples of Poincaré sections for switching-time length Ts = (a) 0.25 , (b) 0.35, (c) 0.50 , (d) 0.75 , (e) 1.00 , and (f) $2.00 \mathrm{~s}$. (g) The result of the $T-D_{2}$ graph and the results of ANOVA and AIC. The black solid line is a fitting curve of $\alpha / T$. 
than the values for $0.50 \mathrm{~s}, 0.35 \mathrm{~s}$, and $0.25 \mathrm{~s}$, and the value for $T=0.75 \mathrm{~s}$ was lower than the values for $0.35 \mathrm{~s}$ and $0.25 \mathrm{~s}$ [where the asterisks in the middle part of Fig. 9(g) show that the relationships between $T$ s were significant]. This result suggests that the values of the correlation dimension increase gradually as $T$ reduces from $2.00 \mathrm{~s}$ to $0.35 \mathrm{~s}$, while the values for $T=0.35 \mathrm{~s}$ and $0.25 \mathrm{~s}$ are not different; this numerically expresses the state that humans become less able to track external inputs gradually as $T$ becomes even shorter.

Theoretically, the relationship between the switching-time length $T$ and the correlation dimension $D_{2}$ suggested by Gohara and Okuyama [1999b], Nishikawa and Gohara 2008 is

$$
D=\frac{-\ln N}{\lambda T},
$$

where $N$ and $T$ are the number of inputs $(N=2)$ and the switching-time length respectively in this study. We are not able to estimate the value of $\lambda$ because $\lambda$ is the internal parameter in the system. However, assuming that the participants in this study performed similar tracking movements and $\lambda$ is constant, the correlation dimension $D_{2}$ is inversely proportional to the switching time length $T(D \propto \alpha / T)$. Accordingly, we fitted a curve with an inverse proportional function $(y=a / T+b)$ to the values of the correlation dimension for all participants and all $T \mathrm{~s}$, and we calculated AIC to this function. First, we calculated the coefficient $a$, constant term $b$ of the fitting curve, and AIC using the value of the correlation dimension for all participants under six $T_{\mathrm{s}}(42$ data $=7$ participants $\times$ $6 T \mathrm{~s})$. Second, we calculated $a, b$, and AIC again after excluding the values of the correlation dimension under $T=0.25 \mathrm{~s}$. Third, we calculated $a, b$, and AIC again after excluding the values under two $T \mathrm{~s}(0.25 \mathrm{~s}$ and $0.35 \mathrm{~s})$. The line graph in the lower part of Fig. 9(g) provides the results of AIC that we obtained with these procedures. The value of AIC was the smallest when we calculated using the value of the correlation dimension for five $T \mathrm{~s}$, i.e. from $0.35 \mathrm{~s}$ to $2.00 \mathrm{~s}$ [the blue filled circle on the line graph in the lower part of Fig. 9(g)]. The fitting curve was $D_{2}=0.178 / T+0.738$ when the value of the residual sum of squares was the smallest. From the above theoretical analysis, we find that in the case of tracking movements performed by a human that require abrupt switching of several movement patterns, the correlation dimension $D_{2}$ is inversely proportional to only time $T$, as reported in the theoretical analysis by Gohara and Okuyama 1999b], Nishikawa and Gohara [2008], if we assume that $\lambda$ is constant although we are not able to estimate this value of $\lambda$ in this study. These results suggest that the value of the correlation dimension increases gradually as $T$ decreases and that a regular relationship such as an inverse proportion $D_{2} \propto 1 / T$ exists between the switching-time length $T$ and the corre lation dimension $D_{2}$.

\section{Discussion}

Recent studies have confirmed that fractal-like and self-similar structures exist not only in various physical phenomena Arecchi et al., 1986; Constantin et al., 1991; Mestl et al., 1997; Maas et al., 1997; Matias et al., 1997; Schmiegel \& Eckhardt, 1997; Tanii et al., 1999; Tanii et al., 1991] but also in human motor control in cases when we switch several movement patterns rapidly with a particular switching-time length $T$ Yamamoto \& Gohara, 2000] or several Ts Suzuki \& Yamamoto, 2015]. Moreover, we confirmed the inverse proportional relationship between $T$ and the fractal-like feature $D_{2}$ in human pointing movement Hirakawa et al., 2016]. Our purpose was to develop these studies on continuous tracking movements and to examine the regular relationship between $T$ and $D_{2}$, which is estimated by the transition state and which occurs when a human switches several attractors stochastically, based on a theoretical model [Gohara \& Okuyama, 1999b; Nishikawa \& Gohara, 2008].

It is assumed that an attractor underlies a movement pattern in a nonautonomous dynamical system with an external input Gohara \& Okuyama, 1999a]. In this study, we confirmed that a movement in which a human tracks continuously an $\mathrm{LED}$ on $\mathrm{L}$ or $\mathrm{R}$ is the limit-cycle attractor, i.e. the excited attractors $\left(A_{L}\right.$ and $\left.A_{R}\right)$, because we qualitatively verified the trajectories in the hypercylindrical state space $\mathcal{M}$ as a continuous dynamical system (Figs. 3 and (4) and statistically and quantitatively the set $C$ on the Poincare section $\Sigma$ as a discrete dynamical system (Table 1) under the periodic-input condition.

The results for trajectory $\Gamma(C)$ in the hypercylindrical state space $\mathcal{M}$ [Figs. 5 (b) 5 (d) and 6 , for the unfolded state space $\theta-(x, \dot{x})[$ Figs. 5 (e), 5 (f) and 8, and for a MANOVA for set $g(C)$ on the Poincaré section $\Sigma(\theta=2 \pi)$ (Table 2 and Fig. 7) suggest that the output of human movement is 
influenced by second-order sequence effects that are composed of the preceding input and the current input and that these are four clusters, i.e. LL, RL, $\mathrm{LR}$, and RR. Moreover, the results of the averaged trajectories in the unfolded state space $\theta-(x, \dot{x})$ (Fig. 8) suggest that each of the four clusters consists of two subclusters, respectively, and that these are the eight clusters. These results imply that the output patterns of human movement are influenced by third-order sequence effects with the external input. Furthermore, the set $g(C)$ on the Poincaré section $\Sigma(\theta=2 \pi)$ under the switching-input condition was a noninteger value in the upper part of Fig. $9(\mathrm{~g})]$. It is well known that a geometric structure is fractal-like if the value of the correlation dimension is a noninteger [Tufillaro et al., 1992]. This signifies that a fractal-like or self-similar structure underlies the output of human continuous movement when humans switch two movement patterns stochastically and rapidly. In other words, the distribution of the outcome by human movement may not be stochastic (such as a Gaussian distribution) or random (such as a uniform distribution) while there are third-order sequence effects.

These results seem to imply the following. Studies on the traditional dynamical systems theory, e.g. that conducted by Kelso and his colleague Kelso, 1995], enable us to investigate human motor control. In their theoretical framework, it was often difficult to apply their theory to a study on various complex human movements that involve abrupt switching of some attractors, i.e. movement patterns. In real-world phenomena, however, the external input provided from the external environment to humans should be abruptly changed and switched. Hence, in our study, human movement was examined using the theoretical framework of the dissipative and nonautonomous dynamical system proposed by Gohara and Okuyama 1999a, 1999b], Nishikawa and Gohara 2008, 2008. Moreover, in a series of previous researches by Kelso and his colleague Kelso, 1995], which were on the state of switching between two attractors, with the continuous dynamical system, the authors had examined the attractor strength, relaxation time, and so on. Therefore, we expanded their autonomous and continuous dynamical systems theory to suit this study, and we applied the theoretical framework of a nonautonomous and continuous dynamical system excited stochastically with an external temporal input to human movement to quantify the state of switching rapidly and abruptly between two attractors. Validating the regular relationship between the switching-time length and the correlation dimension with the fractals generated by switching the vector fields in the hyper-cylindrical state space $\mathcal{M}$ is significant.

In addition, as the results of the inverse proportional relationship $D \propto 1 / T$ in this study are almost in agreement with the theoretical analysis reported in Gohara \& Okuyama, 1999b; Nishikawa \& Gohara, 2008], it is considered that the correlation dimension $D_{2}$ is inversely proportional to the switching-time length $T$. However, the following findings emerged when we analyzed the relationship. The noninteger values estimated by using $D_{2}$ were different among $T \mathrm{~s}$. The fitting curve estimated by using the ANOVA results of the multiple comparisons [see the asterisks in the middle part of Fig. 2(g)] and AIC [see the lower part of Fig. 9(g)] suggest that a regular relationship of inverse proportion exists between $T$ and $D_{2}$. The correlation dimension, which depends on $T$, can be understood intuitively if we consider both sides of the fitting curve, as Nishikawa and Gohara [2008] proposed. That is, two attractors converge to two regions [for example, set $C$ in Fig. 9(f)] as $T$ becomes longer and longer infinitely $(T \rightarrow \infty)$ and $D_{2}$ approaches zero $\left(D_{2} \rightarrow 0\right)$. In contrast, trajectories $\Gamma(C)$ spread around two attractors on the Poincaré section $\Sigma$ with a rapid transition between attractors while $D_{2}$ increases up to 2 if $T$ approaches zero $(T \rightarrow 0)$. However, the tracking movement with rapid switching did not increase and exceed $D_{2} \approx 1.4$ as $T$ decreased [upper part of Fig. 9(g)]. This result for human movement implies that the values of the correlation dimension do not increase without limit as $T$ decreases and do not exceed a certain noninteger value $\left(D_{2} \approx 1.4\right)$ and possibly remain constant ( $D_{2} \approx 1.2$ for the mean value). In addition, the differences among $T=0.50 \mathrm{~s}, 0.35 \mathrm{~s}$, and $0.25 \mathrm{~s}$ for the multiple comparisons were not significant, suggesting that human movement may not become random no matter how short the switching-time length is. Perhaps the reason might be that human movement is affected by biomechanical and/or neuroanatomical constraints. Therefore, a certain amount of complexity (for example, the constant term of the fitting curve, i.e. 0.738 in this study) is inherent in the output patterns of human movement; the output from human movement is not completely 
regular such as it would be for an industrial robot even if $T$ is long. In addition, the system used by humans for tracking movements in this study might have a state of anomaly with the critical time length Nishikawa \& Gohara, 2008] between the switchingtime lengths $0.35 \mathrm{~s}$ and $0.25 \mathrm{~s}$. That is, the output or the response of the system, i.e. the trajectory set $\Gamma(C)$, forms a hierarchical tree structure, and this might suggest that the fractal transition may have relevancy in information processing, such as in motion control [Gohara \& Okuyama, 1999b]. If we interpret this phenomenon from this point of view, humans seem to switch the feedback system to the feedforward system at this critical time length.

\section{Summary}

This study analyzed the movements of participants who continuously tracked two target LEDs located on the left and the right sides in front of them, i.e. $\mathrm{L}$ and $\mathrm{R}$, as accurately as possible. The experimental setting involved the periodic-input condition, in which the same external input was repeated, and the switching-input condition, in which two external inputs were switched stochastically. Consequently, we confirmed that the tracking movement towards $\mathrm{L}$ and $\mathrm{R}$, which was excited by the external input, consisted of two clearly different attractors. Although this result suggests that the relation for $D_{2} \propto 1 / T$ might, in general, hold for dissipative and continuous dynamical system excited by a temporal input, there is a possibility of anomaly as the switching-time length is reduced. Therefore, we should study motor responses, e.g. the value of $D_{2}$ in Fig. 9(a), from the point of view of anomaly in human movement while calculating the inverse proportional function in the future. However, we also believe and conjecture that such complex dynamics provides an origin of various complex phenomena Nishikawa \& Gohara, 2008] for human movement, and that the results reported in this paper are generally qualitatively applicable to dynamical systems excited by external temporal inputs. The conclusions of this study are as follows. (1) The structure of variability of the transition state between several movement patterns is not composed of stochastic or random errors, such that human motor control can switch several movement patterns abruptly. (2) The structure is fractal-like and/or self-similar, and (3) A regular inversely proportional relationship between the structure $D_{2}$ and the switchingtime length $T$ is possible.

\section{Acknowledgments}

The work has been supported in part by Grantsin-Aid for Scientific Research 20240060, 24240085, 24700629, and 15K01538 from the Japan Society for the Promotion of Science.

\section{References}

Arecchi, F. T., Gadomski, W. \& Meucci, R. [1986] "Generation of chaotic dynamics by feedback on a laser," Phys. Rev. A 34, 1617-1620.

Constantin, P., Procaccia, I. \& Sreenivasan, K. R. [1991] "Fractal geometry of isoscalar surfaces in turbulence: Theory and experiments," Phys. Rev. Lett. 67, 17391742.

Gohara, K. \& Okuyama, A. [1999a] "Dynamical systems excited by temporal inputs: Fractal transition between excited attractors," Fractals 7, 205-220.

Gohara, K. \& Okuyama, A. [1999b] "Fractal transition: Hierarchical structure and noise effect," Fractals 7, 313-326.

Grassberger, P. \& Procaccia, I. [1983] "Characterization of strange attractors," Phys. Rev. Lett. 50, 346-349.

Haken, H., Kelso, J. A. S. \& Bunz, H. [1985] "A theoretical model of phase transitions in human hand movement," Biol. Cybern. 51, 347-356.

Hirakawa, T., Suzuki, H., Okumura, M., Gohara, K. \& Yamamoto, Y. [2016] "Switching dynamics between two movement patterns varies according to time interval," Int. J. Bifurcation and Chaos 26, 1630026-1-18.

Kelso, J. A. S. [1984] "Phase transitions and critical behavior in human bimanual coordination," Am. J. Physiol. 240, R1000-R1004.

Kelso, J. A. S. [1995] Dynamic Pattern: The SelfOrganization of Brain and Behavior (MIT Press, Cambridge).

Kloeden, P. E. \& Pötzsche, C. [2013] "Nonautonomous dynamical systems in the life sciences," Nonautonomous Dynamical Systems in the Life Sciences, eds. Kloeden, P. E. \& Pötzsche, C. (Springer International Publishing), pp. 3-39.

Maas, L. R. M., Benielli, D., Sommeria, J. \& Lam, F. P. A. [1997] "Observation of an internal wave attractor in a confined, stably stratified fluid," Nature $\mathbf{3 8 8}$, $557-561$.

Matias, M. A., Muñuzuri, V. P., Lorenzo, M. N., Mariño, I. P. \& Villar, P. [1997] "Observation of a fast rotating wave in rings of coupled chaotic oscillators," Phys. Rev. Lett. 78, 219-222.

Mestl, T., Bagley, R. J. \& Glass, L. [1997] "Common chaos in arbitrarily complex feedback networks," Phys. Rev. Lett. 79, 653-656.

Nishikawa, J. \& Gohara, K. [2008] "Fractals in an electronic circuit driven by switching inputs," Int. J. Bifurcation and Chaos 12, 827-834. 
Nishikawa, J. \& Gohara, K. [2008] "Anomaly of fractal dimensions observed in stochastically switched systems," Phys. Rev. E 77, 036210.

Oldfield, R. C. [1971] "The assessment and analysis of handedness: The Edinburgh inventory," Neuropsychologia 9, 97-113.

Sato, S. \& Gohara, K. [2001] "Fractal transition in continuous recurrent neural networks," Int. J. Bifurcation and Chaos 11, 421-434.

Schmiegel, A. \& Eckhardt, B. [1997] "Fractal stability border in plane couette flow," Phys. Rev. Lett. 79, $5250-5253$.

Schöner, G. \& Kelso, J. A. S. [1988] "A synergetic theory of environmientally-specified and learned patterns of movement coordination," Biol. Cybern. 58, 71-80.

Strogatz, S. H. [2001] Nonlinear Dynamics and Chaos (Westview Press, NY).

Suzuki, H. \& Yamamoto, Y. [2015] "Robustness to temporal constraint explains expertise in ball-over-net sports," Hum. Mov. Sci. 41, 193-206.

Tanii, K., Tachikawa, M., Tohei, T., Hong, F. L. \& Shimizu, T. [1991] "Instability and chaos in two-mode oscillation of a $\mathrm{CO}_{2}$ laser modulated by a saturable absorber," Phys. Rev. A 43, 1498-1501.

Tanii, K., Tohei, T., Sugawara, T., Tachikawa, M. \& Shimizu, T. [1999] "Two different routes to chaos in a two-mode $\mathrm{CO}_{2}$ laser with a saturable absorber," Phys. Rev. E 59, 1600-1604.

Tufillaro, N. B., Abbott, T. \& Reilly, J. [1992] An Experimental Approach to Nonlinear Dynamics and Chaos (Addison-Wesley, Redwood City, CA).

Wada, R. \& Gohara, K. [2001a] "Closures of fractal sets in non-linear dynamical systems with switched inputs," Int. J. Bifurcation and Chaos 11, 22052215.

Wada, R. \& Gohara, K. [2001b] "Fractal and closures of linear dynamical systems excited stochastically by temporal inputs," Int. J. Bifurcation and Chaos 11, $755-779$.

Yamamoto, Y. \& Gohara, K. [2000] "Continuous hitting movements modeled from the perspective of dynamical systems with temporal input," Hum. Mov. Sci. 19, 341-371. 